\title{
Performance Evaluation of DS-CDMA System based Orthogonal Chaotic Vectors over Rayleigh Fading Channel
}

\author{
Maher K. Mahmood \\ Professor \\ Dept. of Electrical Engineering \\ Engineering College \\ AL- Mustansiryah University
}

\author{
Besma Nazar Nathem \\ Dept. of Electrical Engineering \\ Engineering College \\ AL- Mustansiryah University
}

\begin{abstract}
Spreading codes with low cross-correlation values are needed to suppress the effect of multiple access interference (MAI). The chaotic system can be used to generate very large number of sequences due to its sensitive property to initial conditions but due to the quasi-orthogonal nature of chaotic sequences the MAI between active users is generated which leads to degrade the performance of the system. Another type of chaotic sequences with zero values of cross-correlation called orthogonal chaotic vectors (OCVs) are generated by using logistic map and the basic idea of Gram-Schmidt orthonormalization process. In this paper, performance of OCVs in DS-CDMA system for transmission data for different active users over different channels (Rayleigh Flat fading channel and Rayleigh Frequency selective channel) is given. Simulation results show that the DS-CDMA based on OCVs gives better BER performance than other systems. The results also show that the system based on OCVs has the same BER performance regardless of the number of users under the effect of flat fading channel. While at $\mathrm{BER}=10^{-3}$, number of active users $=8$ and spread factor 160 there is about $6 \mathrm{~dB}$ performance difference between the system based on OCVs and system based on Non-OCVs under the effect of time invariant frequency selective fading channel and this difference is decreased to $1 \mathrm{~dB}$ when spread factor is 320 .
\end{abstract}

\section{Keywords}

Orthogonal chaotic vectors, Non-Orthogonal chaotic vectors, Gram-Schmidt ortho-normalization process, Fading channel.

\section{INTRODUCTION}

Direct sequence-code division multiple access (DS-CDMA) technique is used in cellular communication systems where users in the cell are separated from each other with their individual spreading sequences $[1,2]$. Spreading sequences play an important role in multiple access capacity of DSCDMA system. Conventionally, a pseudo random (PN) sequences, such as (Gold codes ,Kasami sequences ...etc.), generated by linear feedback shift registers (LFSRs) had been used for DS-CDMA communication systems but the PN code has a lot of drawbacks due to the fact that there are limited numbers of available PN sequences and they show periodic correlation properties $[1,2,3,4]$. Application of chaotic waveforms to digital communication is research interest for about two decades and the properties of chaotic waveforms such as wide spectrum, sensitive to initial conditions, impulse like auto correlation function and very low cross correlation values make it very attractive to use as an alternate to conventional spreading sequences in spread spectrum communication in addition, very large number of different sequences can be generated simply by changing its initial condition $[1,2,5,6,7,8]$. This makes the number of available chaotic sequences for DS-CDMA systems be very large and this properties provide advantages to chaotic sequences over the conventional PN sequences based multiple access systems $[1,2]$. Another version of chaotic sequences based DS-CDMA system with zero cross-correlation values can be generated by using the chaotic system and the basic idea of the GramSchmidt ortho-normalization process and those sequences are called Orthogonal Chaotic Vectors (OCVs). The use of OCVs in DS-CDMA system leads to suppress the effect of cochannel interference between users and enhance system performance due to zero values cross-correlation of these sequences. The application of orthogonal chaotic vector for multi-level chaotic communication has been previously discussed in $[9,10]$.In this paper performance of OCVs in multiuser chaos based DS-CDMA system over fading channel is given. At the receiver side a simple active correlator type is used for demodulation ,perfect channel estimation for the case of transmitted over flat fading channel is assumed and in order to simplify the analysis of DS-CDMA system detector, the synchronous assumption at the receiver side is made.

The rest of the paper is organized as follows: Section 2 introduces logistic map and their properties. Section 3 describes the generation of OCVs by using Gram-Schmidt ortho-normalization process. Computer simulation is presented in section 4. Finally, the simulation results and conclusions are introduced in sections 5 and 6 respectively.

\section{LOGISTIC MAP}

The logistic map is given by the following equation [11]

$\mathrm{x}(\mathrm{k}+1)=\mu \mathrm{x}(\mathrm{k})(1-\mathrm{x}(\mathrm{k}))$

Where $\mu$ is a constant called the bifurcation parameter $[\mu \in(0,4)]$ and $[x \in(0,1)]$. The equation of logistic map is a key example that shows behavior that is periodic, stable, and chaotic and its behavior depends on the value of $\mu$. For $\mu \in(3.57,4]$ the sequence generated from this map is chaotic $[11,12]$. The time series plot for this map at $\mu=4$ and $\mathrm{x}_{0}=0.1$ is shown in Figure 1.The concept of sensitivity of logistic map to initial conditions is shown in Figure 2.This figure shows two such sequences for $\mu=4$ with $x_{0}=0.1$ and 0.100001 respectively. The small difference leads to different waveforms after few iterations. The qualitative analysis of logistic map can be carried out by the study of its bifurcation diagram. The bifurcation diagram represents the asymptotic behavior of a discrete dynamical system [13]. It is can be described as a record of change in the behavior of a dynamical system as parameter changes [14] .Figure 3 shows the 
bifurcation diagram of the logistic map plotted for $\mathrm{x}_{0}=0.5$ and different values of $\mu$.From a detailed analysis of this diagram one can conclude that the logistic map has a stable fixed point equal to 0 for $\mu \in(0,1)$ and equal to $\frac{\mu-1}{\mu}$ for $\mu \in(1,3)$.The periodic attractors of period $2^{\mathrm{k}}$ for $\mathrm{k}=1,2, \ldots$ exist for $\mu \in(3,3.57)$ and for $\mu>3.57$ the logistic map has a chaotic behavior [13]. Figure 4 describes the Lyapunov Exponent of the logistic map for bifurcation parameter $\mu$ on the interval [0 4] and from this figure one can note that the chaotic behavior of logistic map occurs when $\mu>3.57$ because of the positive value of the Lyapunov Exponent which indicates the chaotic motion [13].

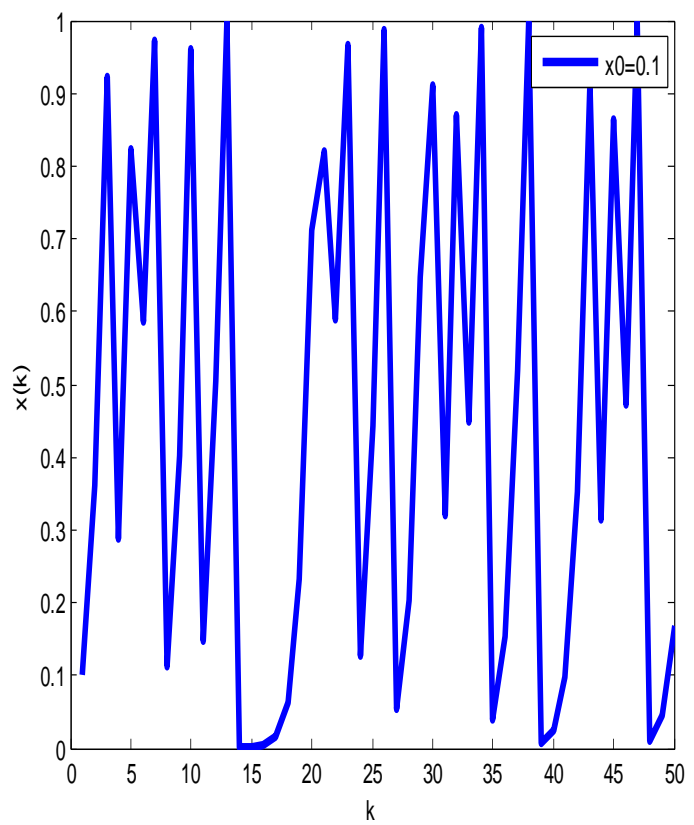

Fig. 1. The time series plot for the logistic map

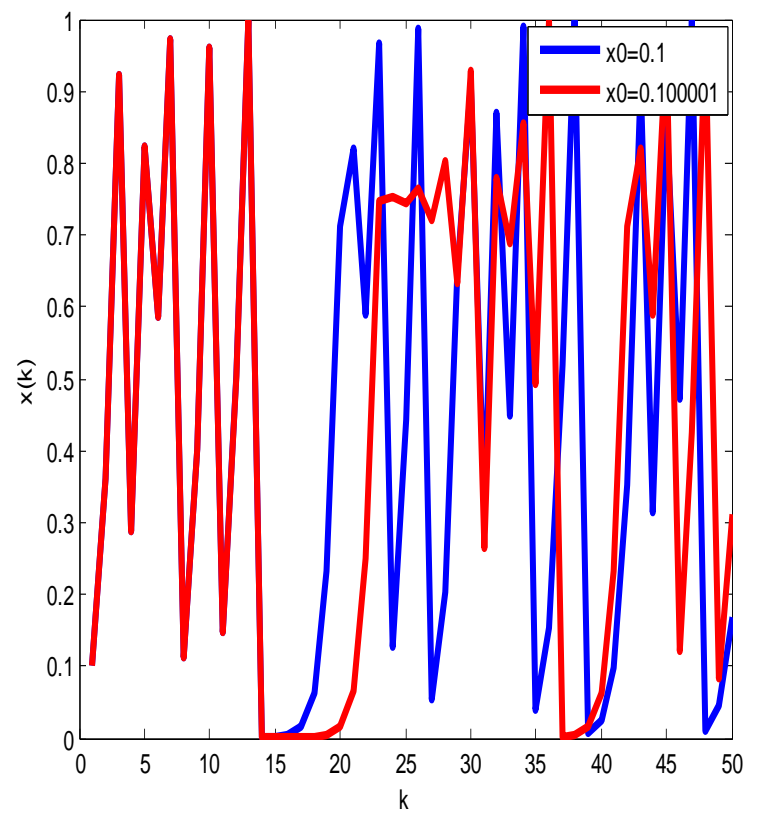

Fig. 2. Chaotic sequence generated for different initial conditions for the logistic map

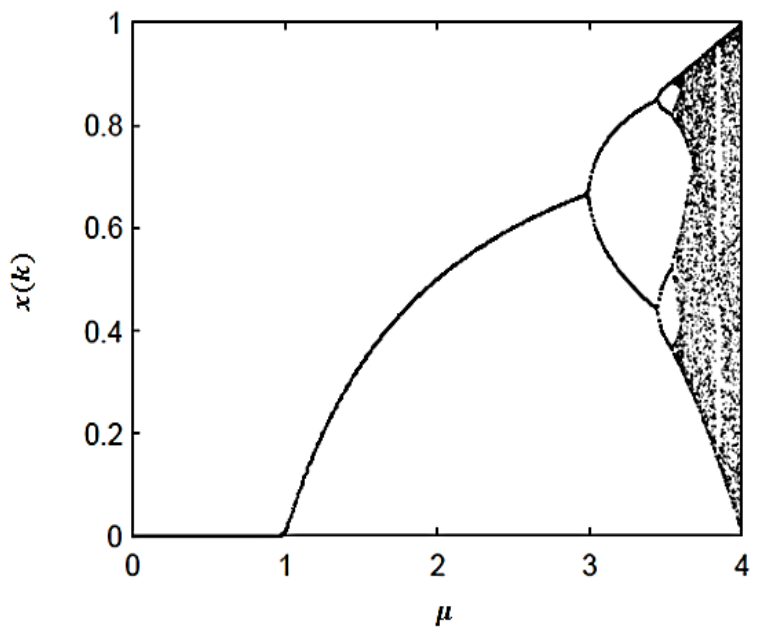

Fig. 3. The bifurcation diagram of the logistic map

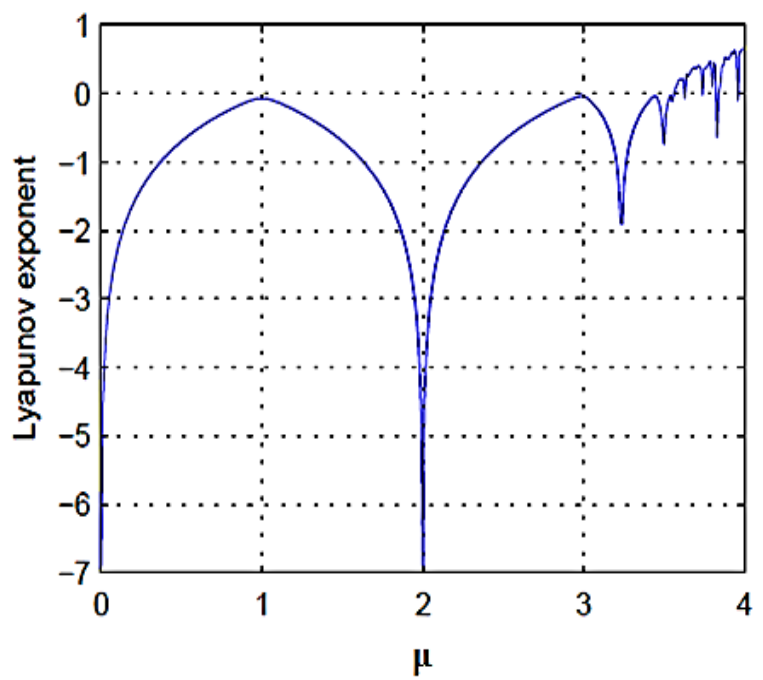

Fig. 4. The Lyapunov Exponent for the logistic map

\section{GENERATION OF ORTHOGONAL CHAOTIC VECTORE (OCVs)}

For DS-CDMA system, the orthogonal chaotic vectors (OCVs) can be generated by using Gram - Schmidt orthonormalization process in order to make the multiple access interference of the $\mathrm{N}_{\mathrm{u}}-1$ interfering signals equal to zero. Figure 5 shows the block diagram that describes the steps of generate OCVs for $\mathrm{N}_{\mathrm{u}}$ active users by using Gram- Schmidt process.

At first step and in order to generate $\mathrm{N}_{\mathrm{u}}$ different chaotic vectors, $\quad \tilde{\mathrm{X}}(\mathrm{k})=\left\{\tilde{\mathrm{x}}(\mathrm{k})^{(1)}, \tilde{\mathrm{x}}(\mathrm{k})^{(2)}, \cdots, \tilde{\mathrm{x}}(\mathrm{k})^{\left(\mathrm{N}_{\mathrm{u}}\right)}\right\}, \quad$ for $\mathrm{N}_{\mathrm{u}}$ different actives users, the same chaotic map with different initial conditions for each active user is used due to their sensitivity to initial conditions. The mean value of each chaotic vector is made zero in order to avoid transmitting $\mathrm{dc}$ power components [5]. Then, a set of $\mathrm{N}_{\mathrm{u}}$ orthogonal chaotic sequences $\tilde{\widetilde{X}}(\mathrm{k})=\left\{\tilde{\tilde{\mathrm{x}}}(\mathrm{k})^{(1)}, \tilde{\mathrm{x}}(\mathrm{k})^{(2)}, \cdots, \tilde{\tilde{\mathrm{x}}}(\mathrm{k})^{\left(\mathrm{N}_{\mathrm{u}}\right)}\right\}$, can be obtained from a set of $N_{u}$ chaotic vectors, $\widetilde{X}(k)$, by using the idea of the Gram-Schmidt ortho-normalization process so that the set of orthogonal chaotic sequences is obtained as follows[5,15]: 


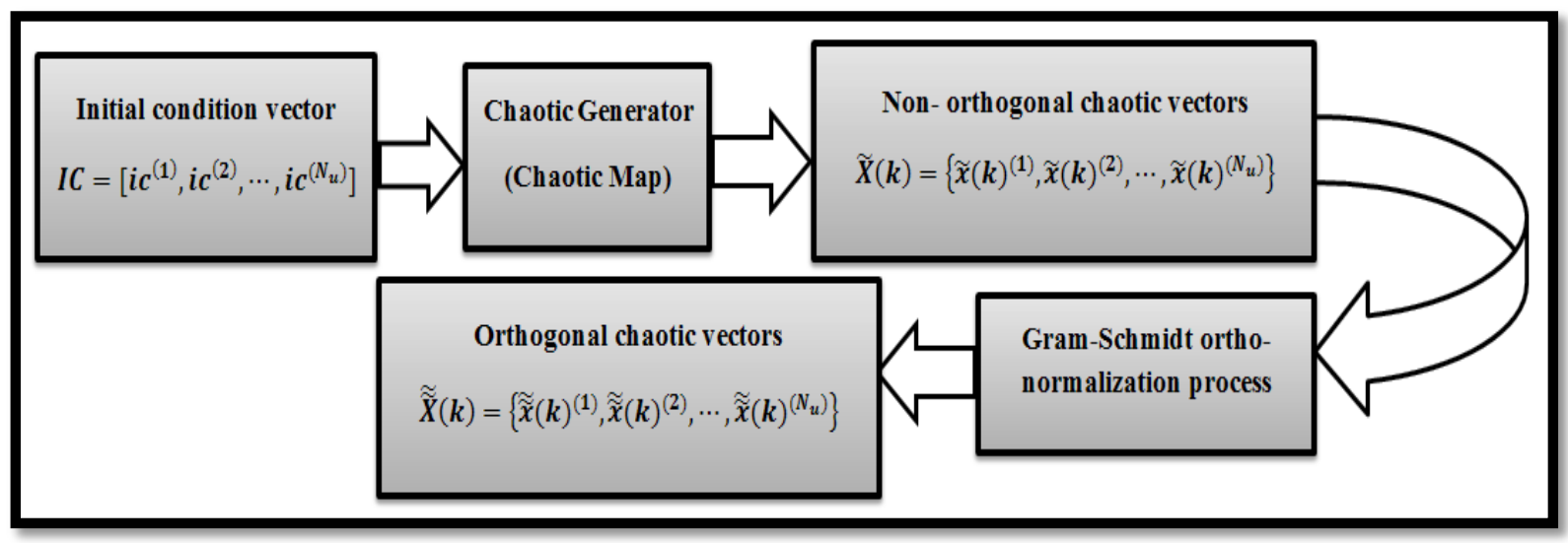

Fig. 5. Orthogonal chaotic vectors Generator

$$
\begin{aligned}
& \tilde{\widetilde{X}}(\mathrm{k})^{(1)}=\frac{\tilde{\mathrm{x}}(\mathrm{k})^{(1)}}{\sqrt{\sum_{\mathrm{k}=1}^{\beta}\left[\tilde{\mathrm{x}}(\mathrm{k})^{(1)}\right]^{2}}} \\
& \tilde{\tilde{\mathrm{x}}}(\mathrm{k})^{(2)}=\frac{\tilde{\mathrm{x}}(\mathrm{k})^{(2)}-\left[\sum_{\mathrm{k}=1}^{\beta} \tilde{\mathrm{x}}(\mathrm{k})^{(2)} \tilde{\mathrm{x}}(\mathrm{k})^{(1)}\right] \tilde{\mathrm{x}}(\mathrm{k})^{(1)}}{\sqrt{\sum_{\mathrm{k}=1}^{\beta}\left[\tilde{\mathrm{x}}(\mathrm{k})^{(2)}-\left[\sum_{\mathrm{k}=1}^{\beta} \tilde{\mathrm{x}}(\mathrm{k})^{(2)} \tilde{\mathrm{x}}(\mathrm{k})^{(1)}\right] \tilde{\mathrm{x}}(\mathrm{k})^{(1)}\right]^{2}}} \\
& \tilde{\tilde{\mathrm{x}}}(\mathrm{k})^{(3)}= \\
& \frac{\check{\mathrm{x}}(\mathrm{k})^{(3)}-\left[\sum_{\mathrm{k}=1}^{\beta} \tilde{\mathrm{x}}(\mathrm{k})^{(3)} \tilde{\mathrm{x}}(\mathrm{k})^{(1)}\right] \tilde{\mathrm{x}}(\mathrm{k})^{(1)}-\left[\sum_{\mathrm{k}=1}^{\beta} \mathrm{x}(\mathrm{k})^{(3)} \tilde{\mathrm{x}}(\mathrm{k})^{(2)}\right] \tilde{\mathrm{x}}(\mathrm{k})^{(2)}}{\sqrt{\sum_{\mathrm{k}=1}^{\beta}\left[\tilde{\mathrm{x}}(\mathrm{k})^{(3)}-\left[\sum_{\mathrm{k}=1}^{\beta} \tilde{\mathrm{x}}(\mathrm{k})^{(3)} \tilde{\mathrm{x}}(\mathrm{k})^{(1)}\right] \tilde{\mathrm{x}}(\mathrm{k})^{(1)}-\left[\sum_{\mathrm{k}=1}^{\beta} \tilde{\mathrm{x}}(\mathrm{k})^{(3)} \tilde{\mathrm{x}}(\mathrm{k})^{(2)}\right] \tilde{\mathrm{x}}(\mathrm{k})^{(2)}\right]^{2}}}
\end{aligned}
$$

And in general, the formula for this process is [5]

$$
\tilde{\tilde{x}}(\mathrm{k})^{(\mathrm{p})}=\frac{\tilde{\mathrm{x}}(\mathrm{k})^{(\mathrm{p})}}{\sqrt{\sum_{\mathrm{k}=1}^{\beta}\left[\tilde{\mathrm{x}}(\mathrm{k})^{(\mathrm{p})}\right]^{2}}} \text { For } \mathrm{p}=1
$$

And

$$
\tilde{\tilde{\mathrm{x}}}(\mathrm{k})^{(\mathrm{p})}=\frac{\tilde{\mathrm{x}}(\mathrm{k})^{(\mathrm{p})}-\sum_{\mathrm{q}=1}^{\mathrm{p}-1}\left[\sum_{\mathrm{k}=1}^{\beta} \tilde{\mathrm{x}}(\mathrm{k})^{(\mathrm{p})} \tilde{\tilde{\mathrm{x}}}(\mathrm{k})^{(\mathrm{q})}\right] \tilde{\mathrm{x}}(\mathrm{k})^{(\mathrm{q})}}{\sqrt{\sum_{\mathrm{k}=1}^{\beta}\left[\tilde{\mathrm{x}}(\mathrm{k})^{(\mathrm{p})}-\sum_{\mathrm{q}=1}^{\mathrm{p}-1}\left[\sum_{\mathrm{k}=1}^{\beta} \tilde{\mathrm{x}}(\mathrm{k})^{(\mathrm{p})} \tilde{\mathrm{x}}(\mathrm{k})^{(\mathrm{q})}\right] \tilde{\mathrm{x}}(\mathrm{k})^{(\mathrm{q})}\right]^{2}}} \quad \text { For } \mathrm{p}=2 \text {, }
$$

$3, \ldots, \mathrm{N}_{\mathrm{u}}$

Where $\tilde{\mathrm{x}}(\mathrm{k})^{(\mathrm{i})}$ and $\tilde{\mathrm{x}}(\mathrm{k})^{(\mathrm{i})}$ are the orthogonal chaotic vector and the chaotic vector for $\mathrm{i}^{\text {th }}$ user respectively, $\mathrm{N}_{\mathrm{u}}$ is the number of active users and $\beta$ represents the number of chaos samples transmitted during one bit duration and is defined as spreading factor.

\section{COMPUTER SIMULATION}

Computer simulations are carried out to evaluate the Bit Error Rate (BER) performance of synchronous DS-CDMA system under the effect of Rayleigh Flat fading channel and Rayleigh Frequency selective channel. Logistic Map which was given in Equations (1) is used in this paper in order to generate the chaotic sequences with different initial conditions assigned to $\mathrm{N}_{\mathrm{u}}$ different active users to generate different chaotic signals.

At the transmitter 10,000 bits are sent from each user. Then, the number of erroneous bits received by each user are noted and divided by the total number of transmitted bits to calculate the BER per user. For simulation purposes and in order to keep the bit energy of the transmitted signal for each user constant, the non-orthogonal chaotic sequences used in DS-CDMA communication system as spreading sequences

\begin{tabular}{|c|c|}
\hline Parameter & Value / Type \\
\hline $\begin{array}{l}\text { No. of transmitted } \\
\text { bits }\end{array}$ & 10,000 bits \\
\hline Data rate & $192 \mathrm{kbps}$ \\
\hline Chip rate & $\beta *$ data rate \\
\hline Doppler frequency & $40 \mathrm{HZ}$ \\
\hline Spreading code & $\begin{array}{l}\text { OCVs, Non-OCVs, Gold } \\
\text { codes, Kasami codes }\end{array}$ \\
\hline Modulation & BPSK \\
\hline Detection & Active Simple Correlator \\
\hline Channel used & $\begin{array}{c}\text { flat fading channel, frequency } \\
\text { selective channel }\end{array}$ \\
\hline $\begin{array}{l}\text { Propagation fading } \\
\text { channel model }\end{array}$ & $\begin{array}{l}\text { Rayleigh fading channel,Jakes } \\
\text { model }\end{array}$ \\
\hline $\mathrm{Eb} / \mathrm{No}$ & $(0-30) \mathrm{dB}$ for fading channel \\
\hline
\end{tabular}
are normalized before modulation. For the purpose of comparison, the system parameters are shown in Table 1.

The software program that has been used for computer simulation is MATLAB 7.12.0 (R2011a).
Table 1. System Parameters

OCVs: Orthogonal Chaotic Vectors, Non-OCVs: NonOrthogonal Chaotic Vectors, BPSK: Binary Phase-Shift-Keying system

\section{SIMULATION RESULTS AND DISSCUTIONS}

The simulated BERs performance of multiuser DS-CDMA communication system which uses OCVs, Non-OCVs and conventional spreading sequences as spreading codes under the effect of Flat fading channel and Frequency selective channel are introduced in this section as follows:

\subsection{Simulated BERs performance of DS- CDMA system under the effect of flat fading channel}

Under the effect of flat fading channel the following aspects of BER performance evaluation for DS-CDMA system are introduced as follows: 


\subsubsection{Variation of BER verses number of active} users $N_{u}$ for Eb/No $=20 \mathrm{~dB}$ and spread factor $\beta=64$ under the effect of flat fading channel The BER performance of multiuser DS-CDMA communication system verses number of active users $N_{u}$ for $\mathrm{Eb} / \mathrm{No}=20 \mathrm{~dB}$ and spread factor $\beta=64$ under the effect of flat fading channel is shown in Figure 6 .From this figure it can be seen that the BER performance of multiuser DS-CDMA system which uses OCVs as spreading sequences is almost constant and is not affected by increasing the number of users. The system will only suffer from the effect of flat fading channel due to the fact that using OCVs in the system leads to cancelation the effect of MAI between active users since the chaotic sequences for all active users in the system are orthogonal to each other due to the use of the Gram-Schmidt ortho-normalization process. While in the case of using NonOCVs and conventional spreading sequences as spreading codes the performance of the system is affected by the effect of flat fading channel and also by increasing the number of active users, where the increase in the number of active users leads to increase the value of MAI between active users therefore the performance of the system is worsen with the increase of the number of active users. One can also note from this figure that because of the good cross-correlation property for small set of Kasami sequences the performance of the system that uses these sequences is better than the performance of the system that uses Non-OCVs, Gold codes and large set Kasami sequences and approaches the performance of the system which uses OCVs. But in spite of this, this set cannot be used in practice in order to increase the capacity of system because the number of sequences available in this set is very small compared with the number of sequences that can be generated by using chaotic system.

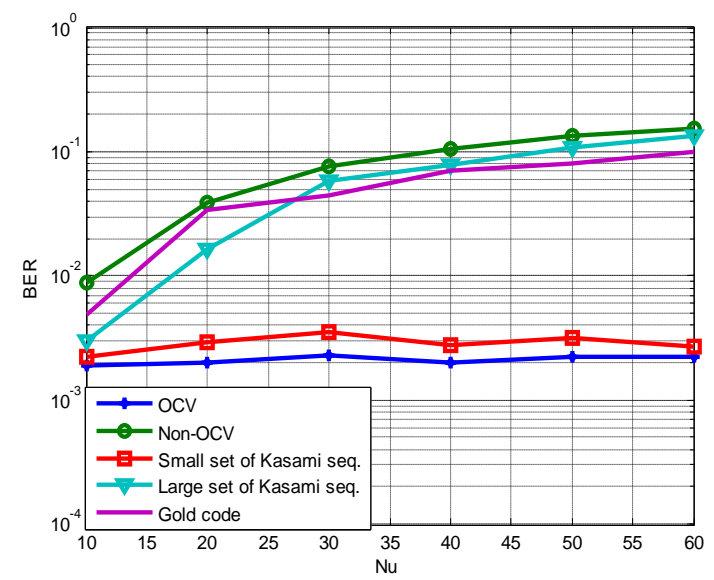

Fig. 6. Variation BER verses number of active users for $\mathrm{Eb} / \mathrm{No}=20 \mathrm{~dB}$ and $\beta=64$ Using OCVs, Non-OCVs and Conventional Spreading Codes under the effect of flat fading channel

\subsubsection{BERs performance of multiuser DS-CDMA} system with OCVs and small set of Kasami sequences for 40 active users and two different values of spread factor $\beta=(50$ and 64$)$ under the effect of flat fading channel

From Figure 7a one can note that the BER performance of DS-CDMA system which uses small set of Kasami sequences approaches the performance of system which uses OCVs for the case of using spreading factor $\beta$ equal to 64 . But when spread factor $\beta$ is decreased to 50 the performance of both systems will diverge from each other and it becomes bad in the system that uses small set of Kasami sequences as shown in Figure $7 \mathrm{~b}$. This is because that the system performance which uses conventional codes to spread the data bits is affected by spread factor $\beta$ and to improve the system's performance, the spread factor must be increased. While in the case of using OCVs there is no need to increase $\beta$ and in order to enhance the system's performance, it is enough that $\beta$ is greater than the number of active users to ensure the orthogonality between the chaotic sequences after using the Gram-Schmidt ortho-normalization process.

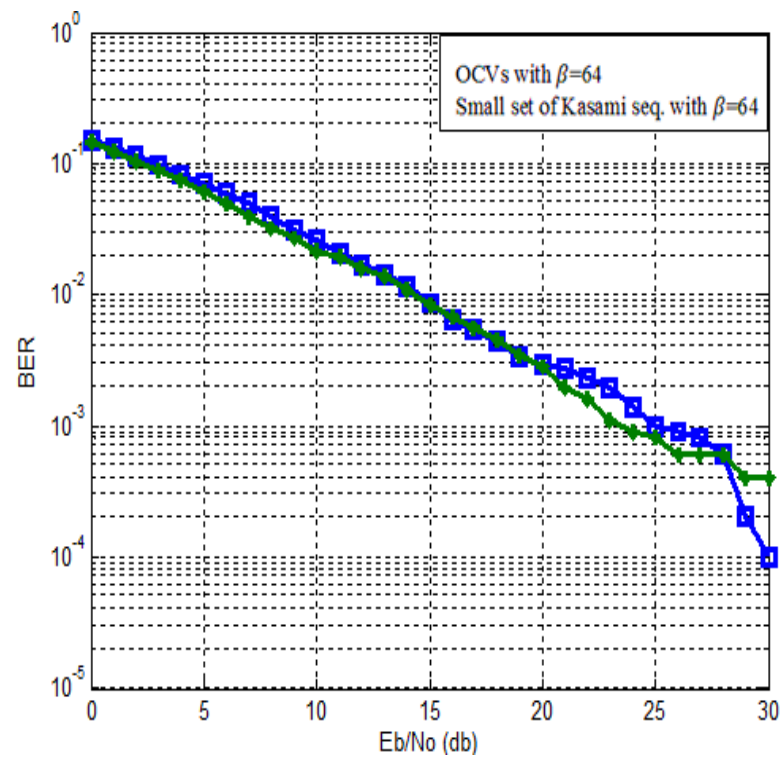

Fig.7a. BER verses $\mathrm{Eb} / \mathrm{No}$ for spread factor $\beta=64$ and number of active users $N_{u}=40$

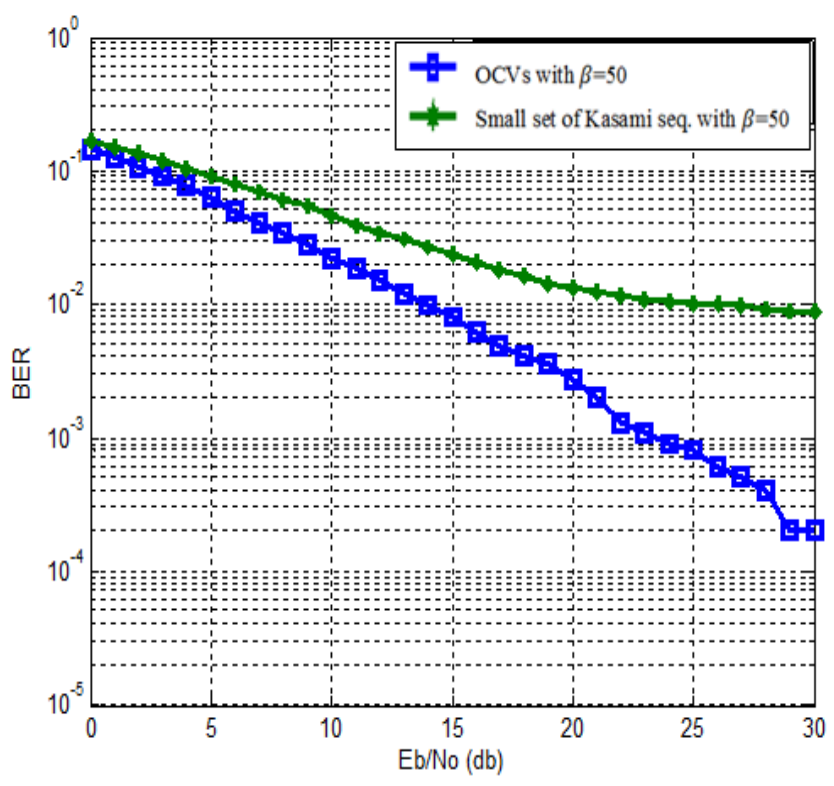

Fig.7b. BER verses $\mathbf{E b} / \mathbf{N o}$ for spread factor $\beta=50$ and number of active users $N_{u}=40$

Fig.7 (a-b). Variation of BER verses Eb/No of DS-CDMA system for number of active $N_{u}=40$ and different values of spread factor using OCVs and small set of Kasami seq. under flat fading channel 


\subsection{Simulated BERs performance of DS- CDMA system under the effect of frequency selective fading channel}

Under the effect of frequency selective fading channel the following aspects of BER performance evaluation for DSCDMA system are introduced as follows:

\subsubsection{BERs performance of multiuser DS-CDMA} system with different spreading codes families and two different values of spread factor under the effect of time varying frequency selective fading channel

The BER performance of multiuser DS-CDMA system verses $\mathrm{Eb} / \mathrm{No}$ with different spreading codes families for 8 active users and two different values of spread factor $\beta$ (40 and 80) under the effect of time varying frequency selective fading channel is shown in Fig.8a and Fig.8b, where the channel's impulse response to any signal transmitted over it will change with time otherwise if the response of the channel does not change with time the fading channel will be called time invariant channel [15].From these figures it can be seen that because of the fast time varying nature of the multipath channel, the multiuser system failed to achieve good BER performance regardless of the type of spreading codes and the values of spread factor $\beta$ where the multiuser DS-CDMA systems have the same BER performance for all different types of codes regardless of the spread factor under the effect of time varying frequency selective channel in the case of using conventional receiver like simple correlator for despreading process. In order to enhance the performance of the system, special type of receiver must be used like Rake receiver that has a bank of correlators that multiplied the received signal with shifted version of local generated codes used at the transmitter side to spread the data bit of active users .To use the Rake receiver, the parameter of channel (delay time, attenuation and phase shift) must be known at the receiver side.

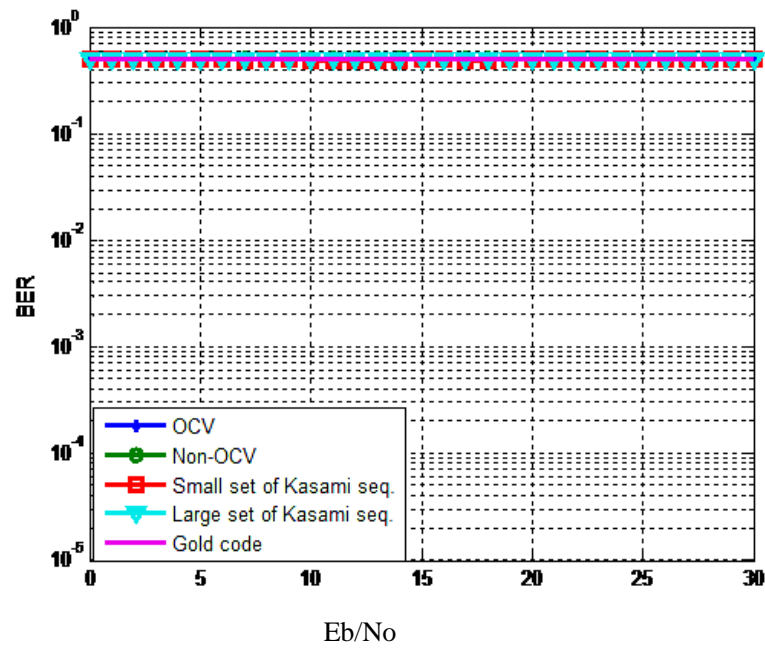

Fig. 8a. BER verses $\mathrm{Eb} / \mathrm{No}$ for spread factor $\beta=40$ and number of active users $N_{u}=8$

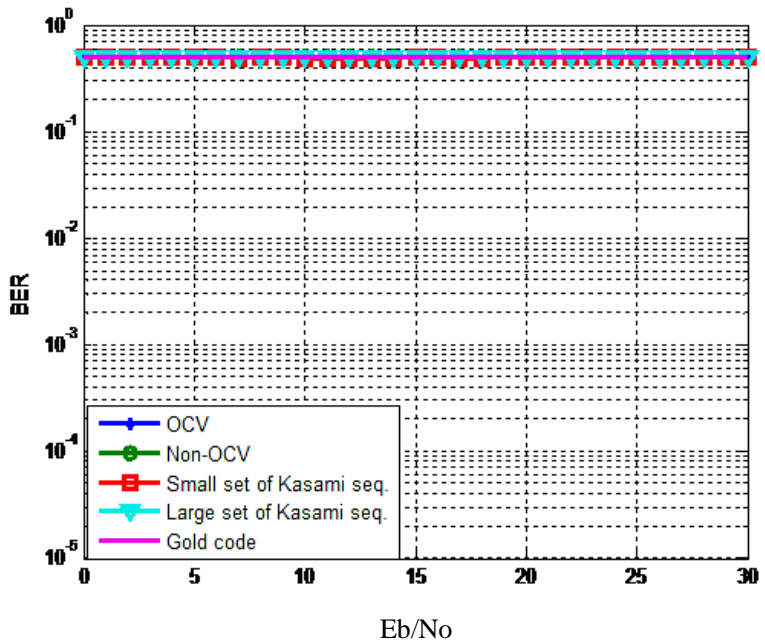

Fig. $8 b$. BER verses $\mathbf{E b} / \mathbf{N o}$ for spread factor $\beta=80$ and number of active users $\mathrm{N}_{\mathrm{u}}=8$

Fig.8 (a-b) . Variation of BER verses Eb/No of DS-CDMA system for number of active users $\mathrm{N}_{\mathrm{u}}=8$ and different values of $\beta$ using OCVs, Non-OCVs and Conventional Spreading Codes under time varying frequency selective fading channel.

5.2.2 BERs performance of multiuser DS-CDMA system with OCVs and Non-OCVs for 8 active users and different values of spread factors $\beta$ under the effect of time invariant frequency selective fading channel

The BER performance of multiuser DS-CDMA system verses $\mathrm{Eb} / \mathrm{No}$ with OCVs and Non-OCVs for 8 active users and different values of spread factors $\beta$ under the effect of time invariant frequency selective fading channel is shown in Figure 9. The channel impulse response for time invariant frequency selective fading is given by [17]:

$\mathrm{h}(\mathrm{k})=0.6742 \delta(\mathrm{k})+0.6030 \delta(\mathrm{k}-1)+0.4264 \delta(\mathrm{k}-2)(7)$

From this figure one can note that under the effect of time invariant frequency selective fading channel, the BER performance of the DS-CDMA system which uses OCVs and Non-OCVs as spreading codes will be improved as the length of spread factor $\beta$ is increased . But in spite of this the BER performance of system which uses OCVs is still better than the performance of other system which uses non-OCVs. This is because that whenever the length of OCVs is greater than the number of active users the MAI between active users is eliminated due to the use of the Gram-Schmidt orthonormalization process and the only factor that affects on the performance of the system will be the multipath interference which is produced due to the multipath nature of the channel and this factor is reduced as spread factor $\beta$ is increased. While the system which uses non-OCVs is affected by two factors, the MAI and the multipath interference. From Fig.9 it can be seen that for 8 users and spread factor $\beta=160$ there is about $6 \mathrm{~dB}$ difference performance at $\mathrm{BER}=10^{-3}$ between the system based on OCVs and system based on Non-OCVs and this difference is decreased to $1 \mathrm{~dB}$ at $\mathrm{BER}=10^{-3}$ between these two systems when the spread factor is increased to 320 . 


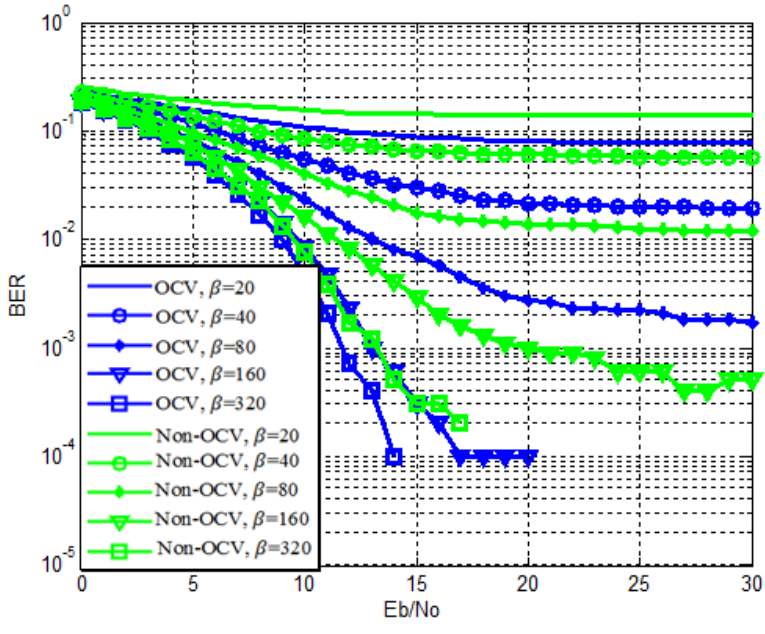

Fig. 9. Variation of BER verses Eb/No of DS-CDMA system for number of active users $\mathrm{N}_{\mathrm{u}}=8$ and different values of $\beta$ using OCVs, Non-OCVs under time invariant frequency selective fading channel.

\subsubsection{Variation of BER verses number of active} users $N_{u}$ for $E b / N o=15 \mathrm{~dB}$ and spread factor $\beta=100$ for multiuser DS-CDMA system under the effect of time invariant frequency selective fading channel

Figure 10 shows the BER performance for DS-CDMA system using OCVs as spreading codes and a comparison of the performance of this system with the performance of the systems that use Non-OCVs and conventional codes like small set of Kasami sequences under the effect of time invariant frequency selective channel at $\mathrm{Eb} / \mathrm{No}=15 \mathrm{~dB}$ for different number of active users and spread factor $\beta$ equal to 100. The same channel impulse response for time invariant frequency selective fading channel which is given in Equation. 7 is used in this section.From this figure one can note that the performance of DS-CDMA based on OCVs is better compared with the rest of the systems that use NonOCVs and small set of Kasami sequences because the orthogonality between the active users codes achieved by using the Gram-Schmidt ortho-normalization process.

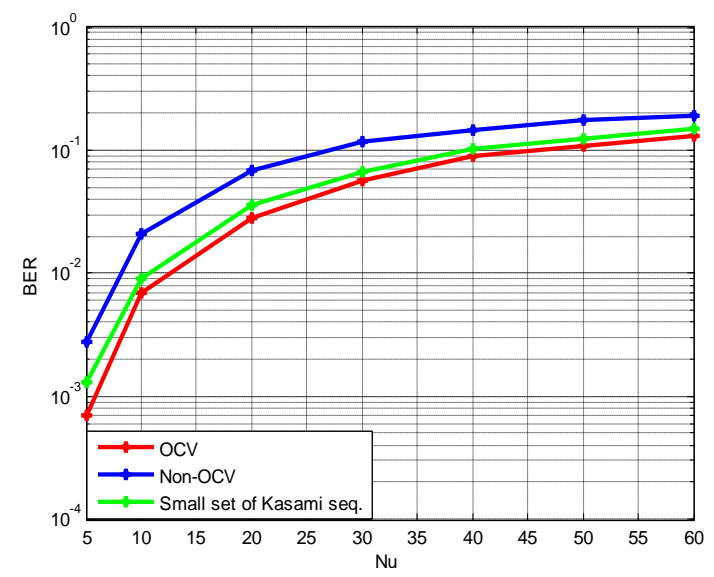

Fig.10. Variation BER verses number of active users for $\mathrm{Eb} / \mathrm{No}=15 \mathrm{~dB}$ and $\beta=100$ using $\mathrm{OCVs}$, Non-OCVs and small set of Kasami sequences under the effect of time invariant frequency selective fading channel.

\section{CONCLUSIONS}

The performance of DS-CDMA depends on using spreading sequences with zero cross correlation value to reduce mutual interference and to make it unaffected by number of user's. In this paper orthogonal chaotic vectors generated using logistic map and Gram-Schmidt ortho-normalization process are used as spreading codes in DS-CDMA system in order to eliminate the effect of MAI. The multiuser DS-CDMA system which uses OCVs gives better BER performance than the other systems which use Non-OCVs and conventional spreading codes (Kasami sequences and Gold codes) under the effect of flat fading channel and its performance will not be decreased as the number of active users in the system is increased. Because the MAI between active users in the system was eliminated due to the use of OCVs as spreading codes and the only factor that affects on the performance of the system is the fading factor due to the nature of the channel. Because of using simple correlator receiver, for de-spreading process the BER performance of DS-CDMA system for all type of spreading codes is the same under the effect of time varying frequency selective channel. To enhance the system's performance and reduce the effect of multipath interference special type of receiver like Rake receiver must be used. Under the effect of time invariant frequency selective channel, the BER performance of DS-CDMA system is getting better as the value of spread factor is increased and the performance of the system which uses OCVs is better than the performance of the system which uses Non-OCVs for the same value of spread factor. At $B E R=10^{-3}$, number of active users $=8$ and spread factor 160 there is about $6 \mathrm{~dB}$ performance difference between the system based on OCVs and system based on Non-OCVs under the effect of time invariant frequency selective fading channel and this difference is decreased to $1 \mathrm{~dB}$ when spread factor is increased to be equal to 320 .

\section{REFERENCES}

[1] L.Bhat and Dr.K.L.Sudha, "Performance Analysis Of Chaotic DS-CDMA With CSK Modulation", International Journal of Mobile Network Communications \& Telematics (IJMNCT), Vol.2, No.2, pp.21-30, April 2012.

[2] L.Bhat and Dr.K.L.Sudha, "Performance Analysis Of Chaotic DS-CDMA With CSK Modulation", International Journal of Advanced Engineering Research and Studies (IJAERS), Vol.1, Iss. 3, pp.133-136,AprilJune 2012.

[3] Sneha Venkateswar, Gargi Rajadhyaksha, and Jinal Shah, "Analysis of Chaotic signals as an alternative Pseudo-Random sequences in DS-CDMA", Canadian Journal on Computing in Mathematics, Natural Sciences, Engineering and Medicine,Vol. 4 No. 2,pp.201-204, April 2013.

[4] Hany A. A. Mansour and Yongqing Fu, “ A New Method for Generating a Zero Mean Self-Balanced Orthogonal Chaotic Spread Spectrum Codes", International Journal of Hybrid Information Technology, Vol.7, No.3, pp.345354,2014

[5] Venkatesh S.and Dr. Poonam S., "Multi User Chaos Based DS-CDMA System Using Orthogonal Chaotic Vector", IEEE, International Conference On Electronics Systems (ICES) NIT Rourkela, pp.339-342, Jan.2011. 
[6] S. O. Starkov and S. V. Yemetz, "Digital Communications Systems, Using Chaos", IEEE, pp.207210, 1997.

[7] G. Kolumbán, M. P. Kennedy, and L. O. Chua, "The Role of Synchronization in Digital Communication Using Chaos-Part I: Fundamentals of digital communications", IEEE Transection on Circuits and Systems -I: Fundamental Theory and Applications, Vol. 44, Iss.10, pp. 927-936, Oct. 1997.

[8] G. Kolumbán, M. P. Kennedy, and L. O. Chua, "The Role of Synchronization in Digital Communication Using Chaos - Part II: Chaotic Modulation and Chaotic Synchronization", IEEE Transection on Circuits and Systems -I: Fundamental Theory and Applications, Vol. 45, Iss.11, pp. 1129-1140, Nov. 1998.

[9] T. J. Wren and T. C. Yang, "Orthogonal Chaotic Vector Shift Keying in Digital Communications", IET Commun., Vol. 4, Iss. 6, pp. 739-753, 2010.

[10] Galias Z., Maggio G.M., "Quadrature chaos-shift keying", IEEE Trans. Syst.-I: Fundam. Theory Appl.,Vol. 48, No. 12, pp. 1510-1519, 2001.

[11] J. B. Seventline, D. E. Rani and K. R. Rajeswari, "Ternary Chaotic Pulse Compression Sequences", Spolecnost pro radioelektronicke inzenyrstvi, Vol. 19, No. 3,pp.415-420, Sep. 2010.
[12] C. Fatima and D. Ali, "New chaotic binary sequences with good correlation property using logistic maps", IOSR Journal of Electronics and Communication Engineering (IOSR-JECE), Vol.5, Iss. 3, pp.59-64, (Mar. - Apr. 2013).

[13] D. Arroyo, G. Alvarez and V. Fernandez, "On the inadequacy of the logistic map for cryptographic applications", Actas De La X Recsi, Salamanca, pp.16,28 May 2008.

[14] AJIDE, O.O, and T.A.O. Salau, "Bifurcation Diagrams of Nonlinear RLC Electrical Circuits", International Journal of Science and Technology (IJST), Vol. 1, No. 3, PP.136-139, Sep. 2011.

[15] Ward C. and David K., "Linear Algebra: Theory and Applications", 1st.India edition, Jones and Bartlett publishers, 2010.

[16] J.G. Proakis , M. Salehi and G. Bauch, "Modren Communication System Using Matlab",3rd. edition, Cengage Learning publisher.

[17] G. Kaddoum, D. Roviras, P. Charge and F.Prunart, "Accurate Bit-Error Rate Calculation for Asynchronous Chaos-Based DS-CDMA over Multipath Channel", EURASIP Journal on Advance in Signal Processing, Vol. 2009,pp.1-12, Jan. 2009. 